Revista Brasileira de Agricultura Irrigada v.10, no.1, p. 410 - 419, 2016

ISSN 1982-7679 (On-line)

Fortaleza, CE, INOVAGRI - http://www.inovagri.org.br

DOI: $10.7127 /$ rbai.v10n100369

Protocolo 369.16 - 05/11/2015 Aprovado em 29/01/2016

\title{
PARAMETRIZAÇÃO DA EQUAÇÃO DE HARGREAVES-SAMANI PARA O ESTADO DO PERNAMBUCO - BRASIL
}

\author{
Francisco D. D. Arraes ${ }^{1}$, Juarez C. Lima Junior ${ }^{2}$, Joaquim B. de Oliveira ${ }^{3}$, Kleber G. de \\ Macedo $^{4}$, Yure de S. Couras ${ }^{5}$, Willame C. de Oliveira ${ }^{6}$
}

\section{RESUMO}

O trabalho tem por objetivo parametrizar o modelo de Hargreaves e Samani para estimativa da evapotranspiração de referência condicionando-o para o nordeste brasileiro utilizando dados meteorológicos de oito cidades do estado do Pernambuco, tomando como referência o modelo de Penman-Monteith. Foram utilizados dados provenientes de estações meteorológicas convencionais de oito municípios: Arcoverde, Cabrobó, Garanhuns, Ouricuri, Petrolina, Recife, Surubim e Triunfo. Os dados meteorológicos foram obtidos junto ao Instituto Nacional de Meteorologia na escala diária e depois transformados na escala mensal, sendo os seguintes dados analisados: velocidade do vento, insolação, temperatura máxima, temperatura mínima, temperatura média, e umidade relativa. Os dois parâmetros empíricos $\alpha$ $(0,0023)$ e $\beta(0,5)$ do modelo foram calibrados de forma simultânea. Para avaliar o desempenho da parametrização foram utilizados os seguintes indicadores estatísticos: índice de Willmott, índice de Nash-Sutcliffe e o erro padrão de estimativa (EPE). Os valores do parâmetro $\alpha$ variaram de 0.000684 (Cabrobó) a 0,0024 (Recife), já $\beta$ de 1,0784 a 0,5 para as mesmas localidades, respectivamente. $\mathrm{O}$ índice de Willmott apresentou valores maiores que 0,90 para todas as estações analisadas, indicando a boa estimativa do modelo calibrado. Já o índice de Nash-Sutcliffe apresentou valores acima de 0,70 mostrando que a calibração da equação de Hargreaves-Samani obteve resultados satisfatórios. O maior valor do EPE foi obtido para a estação localizada em Cabrobó $\left(0,676 \mathrm{~mm} \mathrm{dia}^{-1}\right)$, enquanto que o menor valor foi observado em Surubim com o valor de EPE igual a 0,232 $\mathrm{mm} \mathrm{dia}^{-1}$.

Palavras-chave: Calibração, Evapotranspiração, Penman-Monteith

\section{PARAMETERIZATION OF THE HARGREAVES-SAMANI EQUATION FOR THE STATE OF PERNAMBUCO - BRAZIL}

\footnotetext{
${ }^{1}$ Doutor, Professor do IFSERTÃO - PE/ campus Salgueiro - Pernambuco. Email: dirceuarraes@gmail.com

2 Mestrando em Solos e Nutrição de Plantas, DCS/UFC - Fortaleza - Ceará. Email: junioralencar_ico@hotmail.com

${ }^{3}$ Doutor, Professor do IFCE/ campus Iguatu - Ceará. Email: joaquimbrancodeoliveira@gmail.com

${ }^{4}$ Estudante de Irrigação e Drenagem, IFCE/ campus Iguatu - Ceará. Email:kleber117@hotmail.com

5 Mestrando em Tecnologia e Gestão Ambiental, IFCE/ campus Fortaleza - Ceará. Email:yure.couras@yahoo.com.br

${ }^{6}$ Estudante de Irrigação e Drenagem, IFCE/ campus Iguatu - Ceará. Email: willamecandidoo@gmail.com
} 


\begin{abstract}
The main objective of this study was to parameterize the Hargreaves and Samani equation for the meteorological conditions of the northeastern brazilian region, by using data from eight cities in the state of Pernambuco: Arcoverde, Cabrobó, Garanhuns, Ouricuri, Petrolina, Recife, Surubim and Triunfo. Data were obtained from the National Institute of Meteorology in the daily range and then turned on the monthly scale, with the following data: wind speed, sunshine hour, maximum temperature, minimum temperature, average temperature and relative humidity. The two empirical parameters $\alpha(0.0023)$ and $\beta(0.5)$ were calibrated simultaneously. To evaluate the performance the following statistical indicators were used: Willmott index, Nash-Sutcliffe index and the standard error of estimate (SEE). The $\alpha$ parameter values ranged from 0.000684 (Cabrobó) to 0.0024 (Recife), whereas the $\beta$ parameter values ranged from 1.0784 to 0.5 , respectively, the same cities. Willmott index showed values greater than 0.90 for all analyzed stations, indicating a good e performance of the calibrated equation. But the Nash-Sutcliffe index showed values above 0.70 indicating that the calibration of the Hargreaves and Samani equation achieved a satisfactory performance. The greater SEE value was obtained at the Cabrobó station $\left(0.676 \mathrm{~mm}^{\mathrm{day}}{ }^{-1}\right)$, while the lowest SEE value $\left(0.232 \mathrm{~mm} \mathrm{day}^{-1}\right)$ was observed at Surubim.
\end{abstract}

Keywords: Calibration. Evapotranspiration, Penman-Monteith

\section{INTRODUÇÃO}

De acordo o Senso Agropecuário 2006 (PAULINO et al., 2011, IBGE, 2009), o estado do Pernambuco tem uma área irrigada de 152.917 hectares, sendo o oitavo lugar no ranking dos maiores estados irrigantes do Brasil, com polos importantes de agricultura irrigada, como é o caso do polo PetrolinaJuazeiro (PAULINO et al., 2011). Sendo que em Petrolina estão localizados três perímetros irrigados: Bebedouro, Senador Nilo Coelho e Maria Tereza.

A escassez dos recursos hídricos e a grande demanda de água dos perímetros irrigados do estado do Pernambuco apontam para a necessidade de um manejo adequado da irrigação, que pode ocasionar economia de água e aumento das produções. Uma ferramenta importante para o manejo da irrigação é a estimativa confiável da evapotranspiração de referência (ETo).

A evapotranspiração (ET) é um importante componente do ciclo hidrológico, especialmente para a agricultura irrigada. Os métodos diretos de obtenção da ET são difíceis ou exigem muitas variáveis meteorológicas que nem sempre estão disponíveis em todas as estações meteorológicas. Para suprir essa dificuldade usa-se o conceito de ETo. De acordo com Allen et al. (1998), a ETo expressa a evapotranspiração de uma cultura hipotética de referência com uma altura uniforme de 0,12 $\mathrm{m}$, a resistência de superfície fixa de $70 \mathrm{~s} \mathrm{~m}^{-1}$ e um albedo de 0,23, crescendo ativamente, cobrindo completamente o solo e sem sofrer estresse hídrico. Nessas condições, os únicos fatores que afetam ETo são variáveis climáticas e, consequentemente, a ETo pode ser considerada uma variável atmosférica e pode ser calculada a partir de dados meteorológicos (SENTELHAS et al., 2010).

O modelo FAO Penman-Monteith (PM) tornou-se o padrão aceito para calcular a ETo (PEREIRA et al., 2015). Os dados de entrada necessários para o cálculo da ETo pelo modelo de PM, incluem diversas variáveis climáticas, como temperatura do ar, umidade relativa, velocidade do vento e radiação solar, que nem sempre estão disponíveis, especialmente em países em desenvolvimento (BACHOUR et al., 2013). Quando os dados climáticos são limitados, a equação desenvolvida por Hargreaves e Samani (1985) pode ser utilizada com resultados razoáveis (ALLEN et al., 1998).

A equação de Hargreaves e Samani (HS) exige apenas dados medidos de temperatura do 
ar e radiação extraterrestre (DROOGERS; ALLEN, 2002). Sendo que a mesma tem sido amplamente utilizada por sua simplicidade e potencial para calibrar seus parâmetros e melhorar as estimativas.

Em diferentes regiões do mundo foram encontradas discrepâncias na estimativa da ETo entre os modelos de PM e HS. Sob condições de clima frio e úmido o modelo de HS superestima os valores de ETo quando comparados ao método de PM (TEMESGEN et al., 2005; TRAJKOVIC, 2007), já em locais de clima quente o modelo de HS subestima PM (SIVAPRAKASAM et al., 2011). Portanto, diante do exposto as calibrações locais podem trazer uma melhoria das estimativas da ETo pelo método de HS. Logo, objetivou-se calibrar os coeficientes da equação de HS em escala mensal para oito localidades do estado do Pernambuco.

\section{MATERIAL E MÉTODOS}

Foram utilizados os dados climáticos médios mensais: temperaturas máximas (Tx) e mínimas (Tn) do ar, umidade relativa (UR), velocidade do vento (Vv) e insolação (Ins), oriundos da rede de estações meteorológicas convencionais do Instituto Nacional de Meteorologia (INMET) localizadas em oito cidades do estado do Pernambuco. Na Tabela 1 são apresentadas as localizações das estações: latitude, longitude e altitude bem como os valores médios das velocidades do vento, da umidade do ar e o período de dados disponível.

Tabela 1 - Valores da latitude, longitude, altitude, velocidade do vento média e da umidade relativa média em cada estação.

\begin{tabular}{cccccccc}
\hline ID & Estações & Latitude $\left(^{\circ}\right)$ & Longitude $\left(^{\circ}\right)$ & Altitude(m) & $\begin{array}{c}\mathrm{V}_{\mathrm{v}} \\
\left(\mathrm{m} \mathrm{s}^{-1}\right)\end{array}$ & UR(\%) & Período (anos) \\
\hline 1 & Arcoverde & $-8,41$ & $-37,08$ & 680,70 & 3,75 & 74,17 & $1974 / 2014$ \\
2 & Cabrobó & $-8,51$ & $-39,33$ & 341,46 & 3,71 & 58,69 & $1963 / 2014$ \\
3 & Garanhuns & $-8,88$ & $-36,51$ & 822,76 & 3,57 & 82,24 & $1963 / 2009$ \\
4 & Ouricuri & $-7,90$ & $-40,04$ & 459,28 & 2,87 & 61,49 & $1975 / 2014$ \\
5 & Petrolina & $-9,38$ & $-40,48$ & 370,46 & 3,29 & 55,69 & $1970 / 2014$ \\
6 & Recife & $-8,05$ & $-34,95$ & 10,00 & 2,69 & 78,61 & $1961 / 2014$ \\
7 & Surubim & $-7,83$ & $-35,71$ & 418,32 & 3,90 & 76,17 & $1966 / 2014$ \\
8 & Triunfo & $-7,81$ & $-38,11$ & 1105,00 & 3,18 & 71,99 & $1962 / 2014$ \\
\hline
\end{tabular}

A FAO recomenda que os métodos empíricos devem ser calibrados e validados com base na equação de Penman-Monteith FAO-56 (ALLEN et al., 1998), descrita como:

$$
\text { ETo }=\frac{0,408 \cdot \Delta \cdot\left(R_{n}-G\right)+\gamma \cdot \frac{900}{T m+273} \cdot v_{2}\left(e_{s}-e_{a}\right)}{\Delta+\gamma \cdot\left(1+0,34 \cdot v_{2}\right)}
$$


Em que: ETo é a evapotranspiração de referência em $\mathrm{mm} \mathrm{dia}^{-1}$; $\mathrm{R}_{\mathrm{n}}$ é a radiação líquida total do gramado em $\mathrm{MJ} \mathrm{m}{ }^{-2} \operatorname{dia}^{-1}$; $\mathrm{G}$ é a densidade do fluxo de calor no solo em $\mathrm{MJ} \mathrm{m}^{-2}$ $\operatorname{dia}^{-1}$; Tm é a temperatura média diária do ar em ${ }^{\circ} \mathrm{C} ; \mathrm{v}_{2}$ é a velocidade do vento média diária a 2 $\mathrm{m}$ de altura em $\mathrm{m} \mathrm{s}^{-1}$; $\mathrm{e}_{\mathrm{s}}$ é a pressão de saturação de vapor em $\mathrm{kPa}$; $\mathrm{e}_{\mathrm{a}}$ é a pressão parcial de vapor, $\mathrm{kPa} ; \Delta$ - declividade da curva de pressão de vapor em $\mathrm{kPa} \mathrm{C}^{-1} ; \gamma$ é o coeficiente psicrométrico, $\mathrm{kPa} \mathrm{C}^{-1}$.

$\mathrm{O}$ procedimento de estimativa dos parâmetros para cálculo da evapotranspiração de referência contando apenas com dados de temperatura máxima e mínima do ar segue a metodologia sugerida pelo boletim número 56 da FAO (ALLEN et al., 1998). A estimativa da pressão parcial de vapor $\left(e_{a}\right)$ foi obtida substituindo a temperatura do ponto de orvalho pela temperatura mínima diária do ar, menos $2^{\circ} \mathrm{C}\left(\mathrm{Td}=\mathrm{Tn}-2^{\circ} \mathrm{C}\right)$, conforme sugerido por Allen et al. (1998) para climas semiáridos (Equação 2).

$$
\mathrm{e}_{\mathrm{a}}=0,6018 \cdot \exp \left(\frac{17,27 T_{d}}{T_{d}+237,3}\right)
$$

A radiação solar global $\left(R_{s}\right)$ foi estimada por meio do método de Hargreaves e Samani (1982), (Equação 3).

$$
\mathrm{R}_{s}=K_{r s} \cdot(T x-T n)^{0,5} R_{a}
$$

Em que: $K_{\mathrm{rs}}$ é o coeficiente de ajuste empírico $\left(\mathrm{K}_{\mathrm{rs}}\right)$, o seu valor depende da distância do litoral, sendo o valor de 0,19 para regiões costeiras e 0,16 para regiões continentais; Tx e Tn é a temperatura máxima e mínima do ar, ${ }^{\circ} \mathrm{C}$; Ra é a radiação no topo da atmosfera, $\mathrm{MJ} \mathrm{m}^{-2}$ dia $^{-1}$.

Quando não se dispõem de dados de velocidade do vento $\left(\mathrm{u}_{2}\right)$ registrados, recomenda-se utilizar o valor de $\mathrm{u}_{2}$ médio de 2 $\mathrm{m} \mathrm{s}^{-1}$, conforme descrito por Allen et al. (1998), em função desse número ser uma média de 2000 estações meteorológicas ao redor do globo.
Hargreaves e Samani (1985) propuseram a seguinte equação para estimativa da ETo utilizando apenas os valores das Temperaturas máxima, mínima e média do ar e da radiação no topo da atmosfera:

$$
E T o=\alpha \cdot(T x-T n)^{\beta} \cdot(T m+17,8) \cdot R_{a} \cdot 0,408
$$

Em que: $\alpha$ é um parâmetro empírico, sendo utilizado o seu valor original de 0,0023 ; $\beta$ é um parâmetro empírico exponencial, sendo seu valor original de 0,5 .

As médias mensais de ETo foram calculadas em ambas as metodologias, PM e HS, para todos os locais estudados. As médias mensais de ETo dos meses ímpares foram usados para obtenção dos parâmetros empíricos $(\alpha$ e $\beta)$ e com médias mensais de ETo dos meses pares fez-se a validação do modelo já calibrado pelos parâmetros $(\alpha$ e $\beta$ ) previamente calculados. $\mathrm{O}$ ajuste dos parâmetros da equação de Hargreaves e Samani (1985) foi realizado a partir do Microsoft Excel ${ }^{\circledR}$ utilizando a metodologia descrita por Wraith e Or (1998) para ajuste de equação não linear usando o aplicativo Solver. Essa técnica utiliza como parâmetro de otimização a minimização da soma do desvio ao quadrado (equação 5), ou seja, a diferença entre os valores obtidos por PM e por HS em uma calibração perfeita será próxima a zero.

$$
\sum_{i=1}^{n}\left(P M_{i}-H S_{i}\right)^{2}=0
$$

Em que: $n$ é o número de observações.

A análise dos dados foi realizada de acordo com os índices estatísticos sugeridos por Legates e Mccabe Jr (1999), ou seja, índice de Willmontt (id), coeficiente de Nash-Sutcliffe (E), raiz quadrada do erro médio (RQEM), erro máximo absoluto (EMAX) e a razão entre as médias $(\Gamma)$.

$$
i d=1-\left[\frac{\sum_{i=1}^{n}\left(Y_{i}-X_{i}\right)^{2}}{\sum_{i=1}^{n}\left(\left|Y_{i}-X\right|+\left|X_{i}-X\right|\right)^{2}}\right]
$$




$$
\begin{gathered}
E=1-\frac{\sum_{i=1}^{n}\left(Y_{i}-X_{i}\right)^{2}}{\sum_{i=1}^{n}\left(X_{i}-X\right)^{2}} \\
R Q E M=\sqrt{\frac{\sum_{i=1}^{n}\left(Y_{i}-X_{i}\right)^{2}}{n}} \\
E M A X=\max \{|Y i-X i|\}_{i=i}^{n} \\
\Gamma=\frac{Y}{X} \cdot 100
\end{gathered}
$$

Em que: $\mathrm{X}_{\mathrm{i}}$ é o valor estimado pelo modelo padrão; $\mathrm{Y}_{\mathrm{i}}$ é o valor estimado pelo modelo de HS; $\mathrm{X}$ é o valor da média obtido pelo modelo padrão; Y é o valor da média estimada pelo modelo HS.
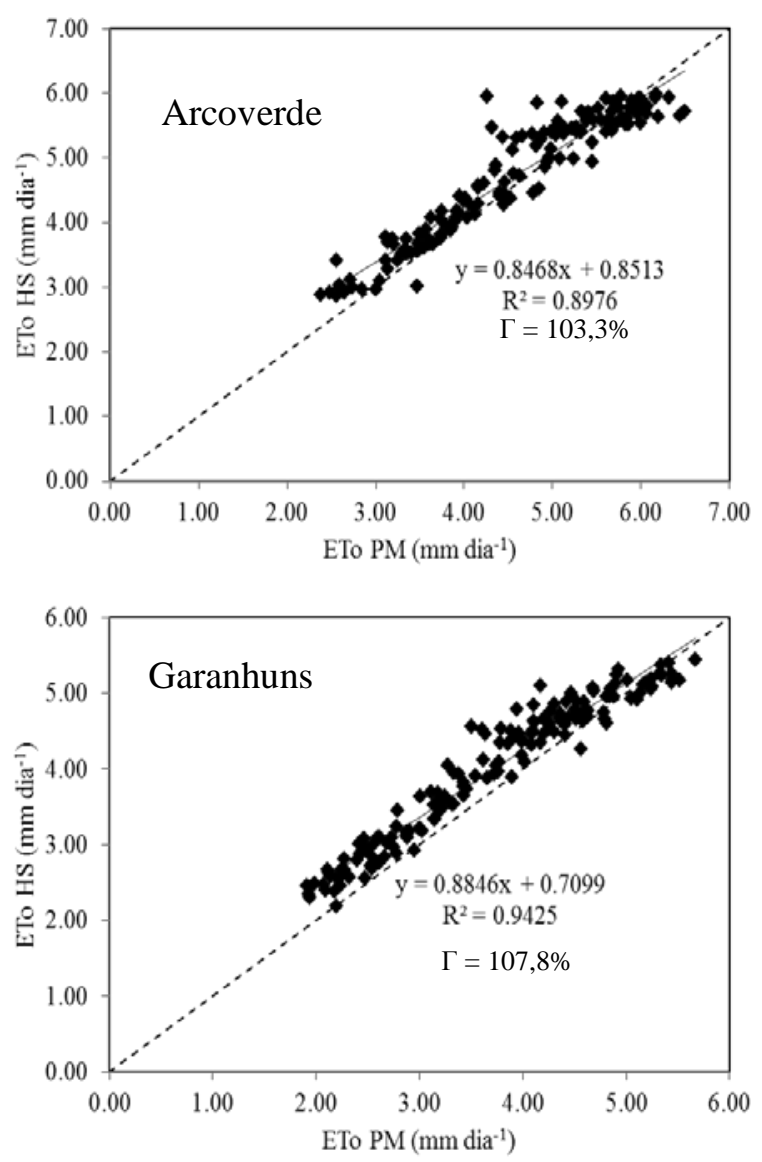

\section{RESULTADOS E DISCUSSÃO}

O desempenho das estimativas da ETo pelo método de HS usando os parâmetros originais ( $\alpha$ igual 0,0023 e $\beta$ igual 0,5 ) pode ser observado nas Figuras 1 e 2. O modelo superestimou os valores da ETo em três das quatro estações que apresentam um valor médio de umidade relativa do ar acima de $70 \%$, as estações de Arcoverde, Garanhuns e Surubim obtiveram valores de $\Gamma$ de $3,3 \%$, $7,8 \%$ e $0,1 \%$ respectivamente (Figuras 1 e 2). Confirmando os resultados obtidos para diversas regiões úmidas do mundo, como por exemplo, o trabalho de Trajkovic (2007) na Servia, Lu et al. (2005) no sudeste dos Estados Unidos da América, Kashyap e Panda (2001) na Índia, Sentelhas et al. (2010) no sul de Ontário Canadá, Saghravani et al. (2009) em Kuala Lumpur na Malásia, Berti et al. (2014) na Itália.
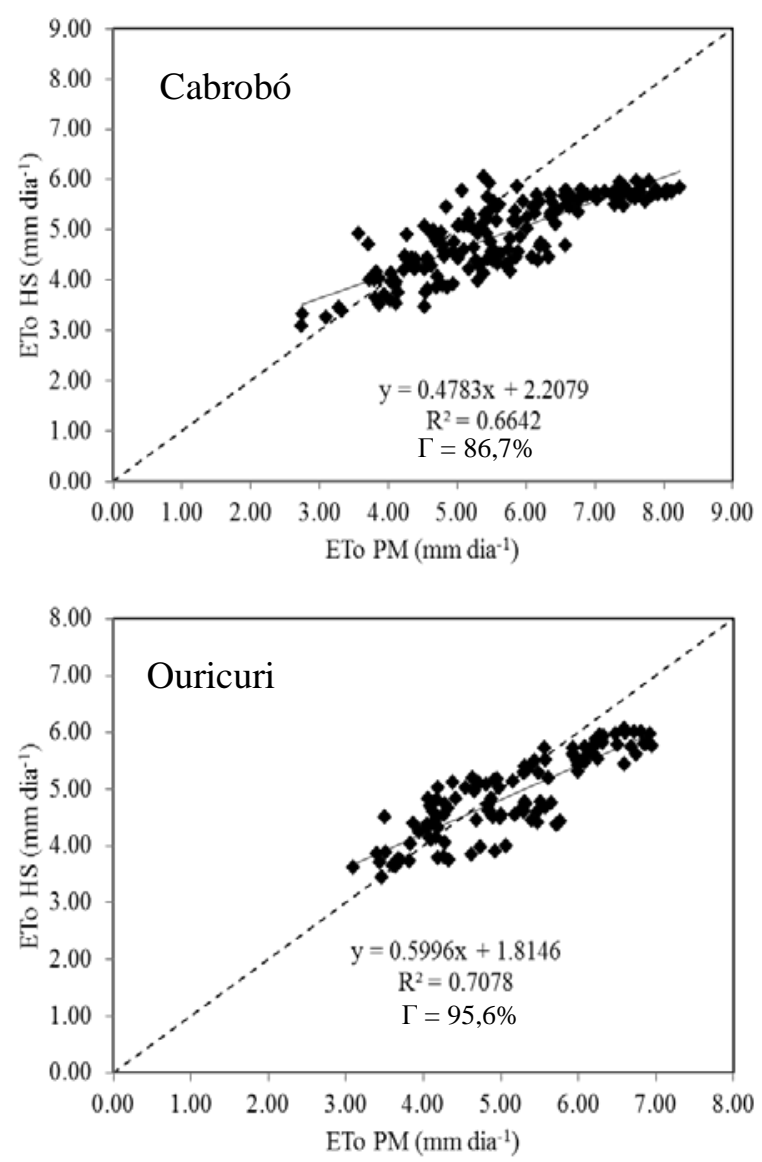

Figura 1 - Comparação entre a ETo obtida pelo método de PM e HS original para as cidades Arcoverde, Cabrobó, Garanhuns e Ouricuri. 

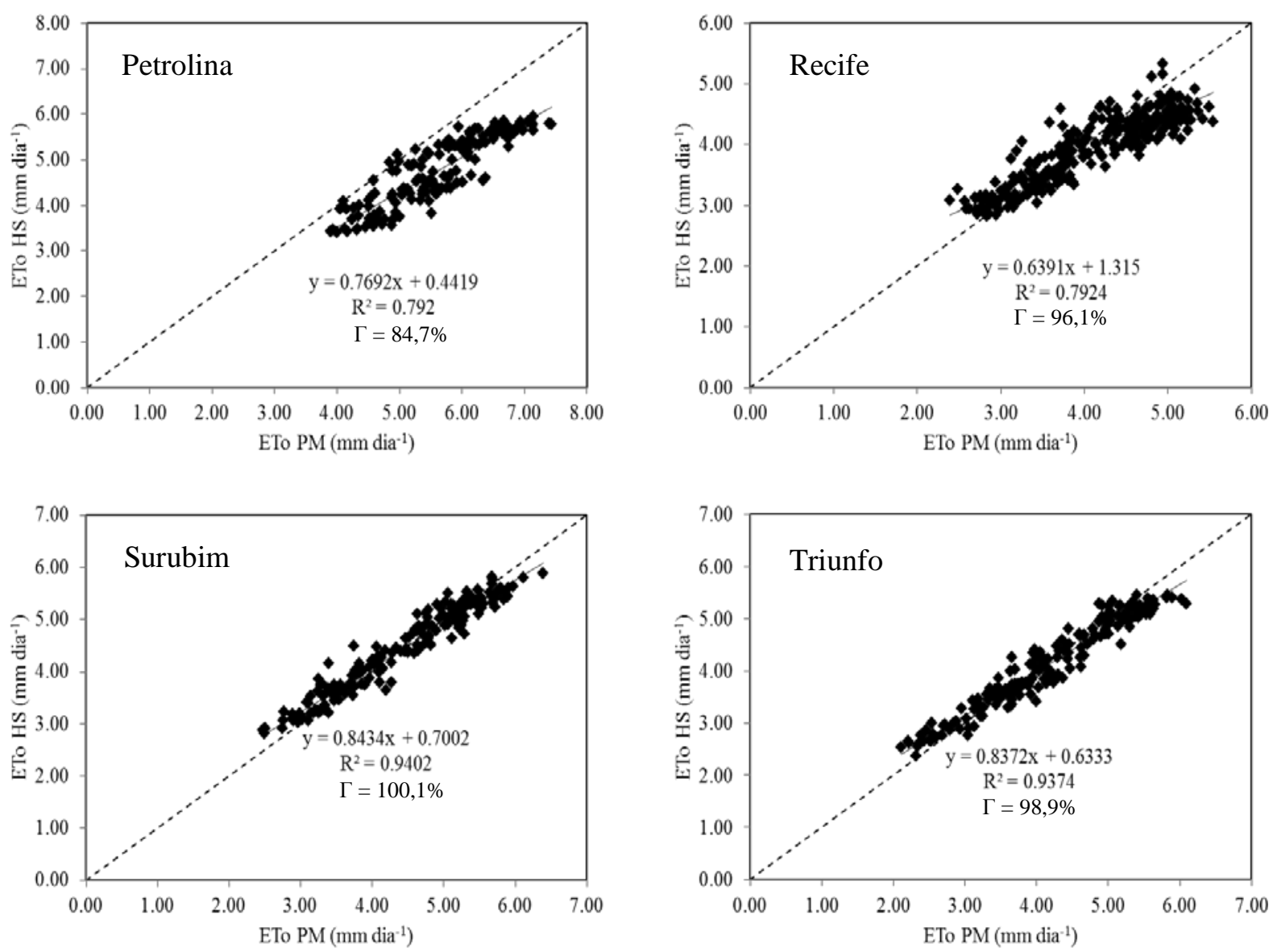

Figura 2 - Comparação entre a ETo obtida pelo método de PM e HS original para as cidades Petrolina, Recife, Surubim e Triunfo.

Nas estações localizadas no sertão pernambucano Cabrobó e Petrolina o método de HS subestimou os valores da ETo em 86,7\% para Cabrobó e 84,7\% para a estação de Petrolina (Figuras 1 e 2). Vale ressaltar que originalmente, o método HS foi desenvolvido para ambientes semiáridos. O comportamento de subestimativa em regiões secas também foi observado por Temesgen et al. (2005) na Califórnia, Khoob (2008) em Mahshad no Irã, Alexandris et al. (2006) em Aquila no EUA.

Na Tabela 2 observa-se o desempenho do modelo original de Hargreaves e Samani para estimativa de ETo das diversas localidades do estado do Pernambuco. Pode-se afirmar de acordo com os valores dos índices estatísticos analisados que o modelo original estima de forma satisfatória a ETo nas localidades que apresentam características de clima mais úmido como é o caso das estações de Arcoverde,
Garanhuns, Recife, Surubim e Triunfo (Tabela 1). Já para as estações localizadas no Sertão (Cabrobó, Ouricuri e Petrolina) o método original não obteve bons resultados de acordo com índices estatísticos utilizados (Tabela 2). O valor de índice eficiência (E) apresentou valor próximo de zero para a estação de Cabrobó e um valor negativo para a estação de Petrolina (Tabela 2). De acordo com Santos (2011), o coeficiente de eficiência (E) representa a razão entre o erro quadrado médio da estimativa e a variância dos dados observados, subtraída da unidade; esse coeficiente varia de $\infty$ a 1 . Valores de $E$ iguais a zero indicam que a média dos dados observados é uma estimativa tão boa quanto os valores preditos pelo modelo; quando $\mathrm{E} \leq 0$, a média dos valores observados é uma estimava melhor do que os valores estimados pelo modelo. Este índice, assim, 
apresenta uma superioridade em relação ao

índice de Willmott em termos de interpretação.

Tabela 2 - Avaliação do desempenho do modelo original de Hargreaves e Samani para estimativa da ETo para as estações localizadas no PE.

\begin{tabular}{ccccccccc}
\hline Índices & Arcoverde & Cabrobó & Garanhuns & Ouricuri & Petrolina & Recife & Surubim & Triunfo \\
\hline Id & 0,965 & 0,732 & 0,962 & 0,873 & 0,736 & 0,903 & 0,980 & 0,978 \\
E & 0,875 & 0,247 & 0,861 & 0,637 & $-0,211$ & 0,721 & 0,930 & 0,924 \\
RQEM & 0,376 & 1,081 & 0,381 & 0,585 & 0,951 & 0,416 & 0,249 & 0,266 \\
Emax & 1,693 & 1,351 & 1,051 & 1,001 & 0,109 & 0,799 & 0,765 & 0,599 \\
\hline
\end{tabular}

O baixo desempenho da equação HS nas estações localizadas no sertão pernambucano ocorre pelo fato da equação de HS não considerar a UR em seu cálculo, sendo que quanto menor a umidade relativa do ar, maior a taxa de evaporação em função do maior déficit de pressão do vapor do ar, portanto, aumentando a demanda evapotranspirométrica (SHAHIDIAN et al., 2013). Os resultados dos índices estatísticos analisados (Tabela 2), para as estações de Cabrobó, Ouricuri e Petrolina, em comparação com as demais estações, justificam a necessidade de calibração do modelo HS para essa região.

O bom desempenho do modelo nas estações mais úmidas deve-se ao fato que nessas localidades ocorre um menor déficit de pressão e valores de velocidades do vento não elevados $\left(<4 \mathrm{~m} \mathrm{~s}^{-1}\right)$, portanto, tem-se que a ETo é uma função apenas da componente da radiação. De acordo com Lemos Filho et al. (2010), existe uma tendência oposta da umidade relativa do ar em relação aos demais elementos meteorológicos, ou seja, locais com registros de maiores valores de umidade relativa do ar (UR) correspondem àqueles onde ocorreram as menores taxas de evapotranspiração, cuja ocorrência se deve ao fato de que a umidade relativa de um local determina o déficit de pressão de vapor que é considerado um indicador da capacidade evaporativa do ar.

Na calibração dos parâmetros da equação de Hargreaves e Samani, a variação do parâmetro $\alpha$ oscilou de 0,00068 (Cabrobó) a 0,00241 (Recife), já o expoente $\beta$ oscilou de 0,50 na estação de Recife, a 1,0784 em Cabrobó (Tabela 3). Ainda de acordo com a Tabela 3, percebe-se que as estações de Arcoverde, Surubim e Triunfo apresentaram valores calibrados muito próximos entre si. Tal resultado pode ser explicado por uma pequena semelhança nas características da umidade relativa do ar e velocidade do vento nessas três estações (Tabela 1 e Tabela 3).

Tabela 3 - Parâmetros ajustados para a equação de Hargreaves e Samani, para cada cidade estudada.

\begin{tabular}{ccccccccc}
\hline \multirow{2}{*}{ Parâmetros } & \multicolumn{7}{c}{ Estações } \\
\cline { 2 - 9 } & Arcoverde & Cabrobó & Garanhuns & Ouricuri & Petrolina & Recife & Surubim & Triunfo \\
\hline$\alpha$ & 0,00142 & 0,00068 & 0,00130 & 0,00078 & 0,00176 & 0,00241 & 0,00160 & 0,00161 \\
$\beta$ & 0,6849 & 1,0784 & 0,7228 & 0,9747 & 0,6796 & 0,5000 & 0,6565 & 0,6545 \\
\hline
\end{tabular}

Os resultados obtidos na calibração equação de Hargreaves e Samani para as diferentes estações meteorológicas pernambucanas apresentaram valores semelhantes aos resultados encontrados por Fernandes et al. (2012), para o estado do Goiás, utilizando dados provenientes de dez estações meteorológicas com intervalo de tempo diferentes entre as estações, obtendo 0,00141 como coeficiente $\alpha$ e 0,68 com o expoente empírico $\beta$, calibrados regionalmente. Os valores médios do parâmetro $\alpha$ e $\beta$ foram 
0,00135 e 0,7439. Já Berti et al. (2014) obteve um valor médio do parâmetro $\alpha$ de 0,0019 e para $\beta$ de 0,517 para dez estações localizadas em uma região de clima úmido na Itália.

Na avaliação da calibração da equação de HS (Tabela 4), apresentou valores do índice de
Willmontt acima de 0,9 em todas as cidades, mostrando uma boa concordância do modelo proposto em relação à equação de PM. Valores semelhantes com os encontrados por Bautista et al. (2009), no México.

Tabela 4 - Avaliação do desempenho do modelo calibrado de Hargreaves e Samani para estimativa da ETo para as estações localizadas no PE.

\begin{tabular}{ccccccccc}
\hline Índices & Arcoverde & Cabrobó & Garanhuns & Ouricuri & Petrolina & Recife & Surubim & Triunfo \\
\hline Id & 0,973 & 0,915 & 0,986 & 0,947 & 0,951 & 0,922 & 0,984 & 0,985 \\
E & 0,897 & 0,706 & 0,946 & 0,804 & 0,793 & 0,772 & 0,938 & 0,942 \\
RQEM & 0,341 & 0,676 & 0,237 & 0,430 & 0,393 & 0,376 & 0,234 & 0,232 \\
Emax & 1,711 & 3,066 & 0,844 & 1,243 & 1,073 & 1,081 & 0,719 & 0,591 \\
\hline
\end{tabular}

Comparando os valores de RQEM presentes na Tabela 2 e na Tabela 4, observa-se que houve uma redução nos valores do RQEM no modelo calibrado para todas as localidades em estudo (Tabela 4). Esse resultado justifica a calibração do modelo HS para estações localizadas no Pernambuco. Ainda de acordo com Tabela 4, tem-se que o RQEM variou entre 0,232 $\mathrm{mm} \mathrm{dia}^{-1}$ (Triunfo) a 0,676 $\mathrm{mm} \mathrm{dia}^{-}$ ${ }^{1}$ (Cabrobó). Shahidian et al (2013) obteve o valor de RQEM de 0,649 $\mathrm{mm}^{\mathrm{dia}^{-1}}$ para as condições climática de Davis, Califórnia. Já Bachour et al. (2013) encontrou valores de RQEM de 0,25 $\mathrm{mm} \mathrm{dia}^{-1}$ para calibração do modelo HS para as condição semiárida no Vale do Bekaa no Líbano.

\section{CONCLUSÃO}

O modelo de Hargreaves e Samani original apresentou resultados insatisfatórios nas estações de Cabrobó, Ouricuri e Petrolina.

A calibração do modelo de Hargreaves e Samani se fez necessária e apresentou um bom desempenho na estimativa da ETo na escala mensal para estações localizadas no Pernambuco. Podendo ser considerada uma alternativa viável para estimativa da ETo em condições de dados limitados.

\section{REFERÊNCIAS BIBLIOGRÁFICAS}

ALEXANDRIS, S.; KERKIDES, P.; LIAKATAS, A. Daily reference evapotranspiration estimates by the "Copais" approach. Agricultural Water Management, v. 82, p. 371-386, 2006.

ALLEN, R. G.; PEREIRA, L. S.; RAES, D.; SMITH, M.. Crop evapotranspiration: guidelines for computing water requeriments. Roma: FAO, 1998. 300 p.

BACHOUR, R. et al. Assessment of Reference Evapotranspiration by the Hargreaves Method in the Bekaa Valley, Lebanon. Journal of Irrigation and Drainage Engineering, v. 139, n. 11, p. 933-938, 2013.

BAUTISTA, F. et al. Calibration of the equations of Hargreaves and Thornthwaite to estimate the potential evapotranspiration in semi-arid and subhumid tropical climates for regional applications. Atmósfera, v. 22, n. 4, p. 331-348, 2009.

BERTI, A. et al. Assessing reference evapotranspiration by the Hargreaves method in north-eastern Italy. Agricultural Water Management, v. 140, p. 20-25, 2014.

DROOGERS, P.; ALLEN, R. G. Estimating reference evapotranspiration under inaccurate data conditions. Irrigation and drainage systems, v. 16, n. 1, p. 33-45, 2002. 
FERNANDES, D.S. et al. Calibração regional e local da equação de Hargreaves para estimativa da evapotranspiração de referência. Revista Ciência Agronômica, v. 43, n. 2, p. 246-255, 2012.

FOOLADMAND, H.R.; HAGHIGHAT, M. Spatial and temporal calibration of Hargreaves equation for calculating monthly ETo based on Penman-Monteith method. Irrigation and Drainage, v. 56, p 439-444, 2007.

HARGREAVES, G. H.; SAMANI, Z. A. Reference crop evapotranspiration from temperature. Applied Engineering in Agriculture, v. 01, n. 02, p. 96-99, 1985.

INSTITUTO BRASILEIRO DE GEOGRAFIA E ESTATÍSTICA. Censo agropecuário - 2006. Rio de Janeiro, 2009.

KASHYAP, P, PANDA, R. Evaluation of evapotranspiration estimation methods and development of crop-coefficients for potato crop in a sub humid region. Agricultural Water Management, v. 50, p. 9-25, 2001.

KHOOB, A. R. Comparative study of Hargreaves's and artificial neural network's methodologies in estimating reference evapotranspiration in a semiarid environment. Irrigation Science, v. 26, n. 3, p. 253-259, 2008.

LEGATES, D. R.; MCCABE, G. J. Evaluating the use of "goodness-of-fit" measures in hydrologic and hydro climatic model validation. Water Resources Research. v. 35, n. 1, p. 233-241, 1999.

LU, J, SUN, G, MCNULTY, S, AMATYA, D. A. Comparison of six potential evapotranspiration methods for regional use in the Southeastern United States. Journal of the American Water Resources Association, v. 41, n. 3, p.621-633, 2005.
PAULINO, J., FOLEGATTI, M. V., ZOLIN, C. A., ROMÁN, R. M. S., JOSÉ, J. V. Situação da agricultura irrigada no Brasil de acordo com o censo agropecuário 2006. Irriga, v. 16, n. 2, p. 163, 2011.

PEREIRA, Luis S. et al. Crop evapotranspiration estimation with FAO56: Past and future. Agricultural Water Management, v. 147, p. 4-20, 2015.

SAGHRAVANI, S, MUSTAPHA S, IBRAHIM, S, RANDJBARAN, E. Comparison of daily and monthly results of three evapotranspiration models in tropical zone: A case study. American Journal of Environmental Sciences, v. n. 6, p. 698-705, 2009.

SANTOS, M.A. Extração de água do solo por plantas de soja: modelagem hidrofísica e empírica. 2011. 76 p. Dissertação (Mestrado em Física do Ambiente Agrícola) - Escola Superior de Agricultura "Luiz de Queiroz", Universidade de São Paulo, Piracicaba, 2011.

SENTELHAS, P. C.; GILLESPIE, T. J.; SANTOS, E. A. Evaluation of FAO PenmanMonteith and alternative methods for estimating reference evapotranspiration with missing data in Southern Ontario, Canada. Agricultural Water Management, v. 97, n. 5, p. 635-644, 2010.

SHAHIDIAN, S. et al. Parametric calibration of the Hargreaves-Samani equation for use at new locations hydrological processes. Hydrological Process, v. 27, p. 605-616, 2013.

SUBBURAYAN， S.; MURUGAPPAN， A.; MOHAN, S. Modified Hargreaves equation for estimation of ETo in a hot and humid location in Tamilnadu State, India. International Journal of Engineering Science and Technology, v. 3, n. 1, 2011. 
TEMESGEN, B. et al. Comparison of some reference evapotranspiration equations for California. Journal of Irrigation and Drainage Engineering, v. 131, n. 1, p. 73-84, 2005.

THEPADIA, M.; MARTINEZ, C. J. Regional Calibration of Solar Radiation and Reference Evapotranspiration Estimates with Minimal Data in Florida. Journal of Irrigation and Drainage Engineering, v. 138, n. 2, p.
111-119, 2012.

TRAJKOVIC, S. Hargreaves versus PenmanMonteith under humid conditions. Journal of Irrigation and Drainage Engineering, v. 133, n. 1, p. 38-42, 2007.

WRAITH, J. M.; OR, D.. Nonlinear parameter estimation using spreadsheet software. Journal of Natural Resources and Life Sciences Education, v. 27, p. 13-19, 1998. 